\title{
A ENFERMAGEM RECONSTRUINDO SUA PRÁTICA: MAIS QUE UMA CONQUISTA NO PSF ${ }^{1}$
}

Maria Fátima de Sousa²

\section{INTRODUÇÃO}

O processo de construção do Sistema Único de Saúde, regulamentado pela Constituição de 1988 , ao ser desenvolvido sobre os pilares da universalização, da descentralização e da integralidade da assistência, visa reduzir o hiato ainda existente entre os direitos sociais garantidos em lei e a capacidade efetiva de oferta dos serviços públicos de saúde à população brasileira.

O modelo assistencial ainda predominante no país caracteriza-se pela prática "hospitalocêntrica", pelo individualismo, pela utilização irracional dos recursos tecnológicos disponíveis e com baixa resolubilidade. Vem gerando alto grau O que proponho neste texto é uma subversão de valores, onde o carro chefe seja a ética, cuja base se fundamenta: não ter nenhuma família no Brasil sem atenção, sem cuidado à saúde. Este será nosso maior patrimônio social e o nosso maior valor ético, sem os quais , continuaremos devedores destas famílias. de insatisfação para todos os partícipes do processo - gestores, profissionais de saúde e população que utiliza os serviços.

Neste contexto, o Ministério da Saúde assumiu, desde 1991, a implantação do Programa de Agentes Comunitários de Saúde, como transitório a formatação em 1994, do Programa de Saúde da Família - PSF, visando, enquanto estratégia setorial, a reorientação do modelo assistencial brasileiro.

A estratégia do PSF propõe uma nova dinâmica para a estruturação dos serviços de saúde, bem como para a sua relação com a comunidade e entre os diversos níveis de complexidade assistencial. Assume o compromisso de prestar assistência universal, integral, equânime, contínua e, acima de tudo, resolutiva à população, na unidade de saúde e no domicílio, sempre de acordo com as suas reais necessidades, identificando os fatores de risco aos quais ela está exposta e neles intervindo de forma apropriada.

Propõe-se, ainda, a humanizar as práticas de saúde, buscando a satisfação do usuário através do estreito relacionamento dos profissionais com a comunidade e estimulando-a ao reconhecimento da saúde como um direito de cidadania e, portanto, expressão de qualidade de vida.

Nesta perspectiva, o PSF foge da concepção usual dos programas tradicionais concebidos no Ministério da Saúde, por não se tratar de intervenção pontual no tempo e no espaço e tampouco de forma vertical ou paralela às atividades rotineiras dos serviços de saúde. Ao contrário, objetiva a integração e a organização das atividades em um território definido, com o propósito de enfrentar e resolver os problemas identificados, com vistas a mudanças radicais no sistema, de forma articulada e perene.

A efetiva implantação dessa estratégia vem possibilitar a busca da integralidade da assistência e a criação de vínculos de compromisso e de responsabilidade compartilhados entre os serviços de saúde e a população.

Todo o sistema deverá estar estruturado segundo a lógica da estratégia em questão e não

1 As opiniões expressas neste texto são de responsabilidade da autora e não representam necessariamente as da Instituição onde atua.

${ }^{2}$ Enfermeira, Especialista em Saúde Pública, Mestra em Ciências Sociais, assessora especial do Departamento da Atenção Básica, da Secretaria de Políticas de Saúde, do Ministério da Saúde 
apenas as unidades básicas, pois a continuidade da atenção por todo o sistema deve ser garantida, através de um fluxo contínuo setorial, sem que haja solução de descontinuidade nesse processo. A unidade básica de saúde, sob a estratégia da Saúde da Família, deve ser a "porta de entrada do sistema local de saúde", mas a mudança no modelo tradicional exige a integração entre os vários níveis de atenção.

A estratégia de Saúde da Família, por ser um projeto estruturante, deve provocar uma transformação interna do sistema, com vistas à reorganização das ações e serviços de saúde. Esta mudança implica na ruptura da dicotomia entre as ações de saúde pública e a atenção médica individual, assim como, entre as práticas educacionais e assistenciais.

Apesar da experiência de Saúde da Família no Brasil caracterizar-se pela sua jovialidade, já apresenta significativa acumulação de práticas e saberes, apontando para a relevante importância de se promover um permanente intercâmbio técnico e científico entre os atores envolvidos no processo.

Já existe um acúmulo de experiências diversificadas em nível nacional que poderiam fornecer elementos de reflexão àqueles que se propõem a viabilizar o SUS em sua localidade. Há de se considerar que cada uma delas vem-se desenvolvendo em uma conjuntura específica e dificilmente poderia haver uma generalização de sua aplicabilidade. Entretanto, uma análise rigorosa de suas principais conquistas e desafios é extremamente importante para os profissionais envolvidos desde sua formulação, implantação, implementação nos ....... municípios que vêm adotado estas estratégias, como um caminho possível à reversão do modelo assistencial vigente

Em particular, este artigo tem um "ambicioso objetivo": servir de veículo de testemunhos e debate acerca do papel central que os enfermeiros vêm imprimindo ao longo destes 10 anos de construção do PASC/PSF nos diferentes recantos do Brasil

\section{O ENFERMEIRO DE FAMÍLIA: RECONSTRUINDO SUA PRÁTICA}

Ë preciso voltarmos as nossas origens para compreendermos as razões pelas quais a Enfermagem vem assumindo, em linha de frente, o processo de formulação, implantação e implementação do PSF no Brasil. Se analisarmos a prática de enfermagem anterior ao século XIX, nos deparamos com uma prática voltada às famílias sem recursos para cuidar de seus doentes, portanto, uma enfermagem independente da medicina, pois suas ações não pressupunham ordens médicas ou planos terapêuticos e sim uma origem e finalidade de cunho comunitário, religioso. Como afirma Almeida e Rocha (1986) a medicina e a enfermagem tiveram suas origens de forma independentes e por muitos séculos atuaram separadamente. Esta independência começa a reconfigurar-se a partir do final do século XVIII e início do XIX, onde o aparato hospitalar abriga o saber e a prática médica, subordinando os "outros" saberes.

Ë sabido que o ato de internar os doentes para tratamento e assistência nos hospitais, onde concentravam-se os escassos recursos disponíveis, levou a institucionalização da enfermagem hospitalar, prática essa que vem se acelerando nas últimas décadas, como modelo hegemônico, colocando, não só a enfermagem mas todos os profissionais da área de saúde a adotarem um modelo caro, desumano e pouco resolutivo.

Esta trajetória, segundo Novaes (1990), gerou, entre outras coisas, o culto à doença e não à saúde, e a devoção à tecnologia, sob a presunção ilusória de que ela seria o centro de atividades científicas e de assistência à saúde.

Para reversão desse quadro, um dos marcos importantes na pratica da Enfermagem, foi a adoção dos princípios da atenção primária à saúde, cuja imagem-objetivo seria a construção social de saúde para todos. E a configuração deste corpo doutrinário de proposições para a mudança do sistema de saúde brasileiro, foram protagonizados por vários programas, projetos e estratégias, a serem lembrados: Programa de Desenvolvimento Rural Integrado - PDRIS; Programa de Interiorização das Ações e Serviços de Saúde e Saneamento - PIASSS; Programa de Preparação Estratégica do Pessoal da Saúde - PPREPS; Programa Intensivo de Mão-de-Obra - PIPMO, Programa de Localização de Unidades e Serviços de Saúde - PLUS, Programa Nacional de Alimentação e Nutrição - PROMAN; Programa Nacional de Saneamento - PLANASA, Programa Nacional de Serviços Básicos de Saúde - 
PREV-SAÚDE, Programa de Ações Integradas de Saúde - PAIS, posteriormente denominado AIS, Programa de Integração Docente Assistencial Programação e Orçamentação Integrada - POI, Programa de Racionalização Ambulatorial, o Sistema de Assistência Médico-Hospitalar da Previdência Social SAMHPS, ou simplesmente Sistema de AlH, entre outros movimentos de disseminação políticoideológica das propostas de reforma sanitária.

Certamente, não é pretensão deste texto destacar a importância da Enfermagem em cada um dos Programas, Projetos e Estratégias acima mencionados, todavia, destacar sua efetiva participação, como sujeito técnico-político-social, comprometido com os princípios que fundamentam a reforma democrática do sistema de saúde brasileiro.

E as bases desse compromisso, digo, desse projeto de nação, credencia a Enfermagem, mais uma vez, a se colocar "em linha de frente", agora na construção do PACS/PSF. E nesta "nova" missão é imperativo afirmar: a enfermagem brasileira, volta a suas origem, pois nasceu como hoje vem se estruturando, voltada e centrada com, para e na comunidade.

\section{A ENFERMAGEM NO PSF: O REENCONTRO DE MENTES E CORAÇÕES}

Segundo a realidade vivida por quase 15.000 enfermeiros espalhados nos mais diferentes recantos deste imenso país, a operacionalização da estratégia Saúde da Família vem gerando, de fato, um novo modelo de atenção à saúde, configurando-se numa forma inovadora de se fazer saúde, fundamentada em uma nova ética social. E neste horizonte, diz bem Eliane Caminha "A gente não pode mudar tudo, mas pode fazer tudo para mudar (enfermeira do PSF de Olinda-PE,1995).

E os elementos desta mudança devem ser baseados nos princípios da justiça, da igualdade de oportunidades, do exercício diário da democracia, tendo como anseio maior o bem estar social e a defesa da vida do cidadão, através da lógica básica da vigilância à saúde, utilizando o enfoque de risco como método de trabalho e sempre voltado às verdadeiras necessidades da população e à solução de problemas localmente identificados.

Com isso, ela acaba gerando novas práticas setoriais, englobando tanto o trabalho clínico individual, como a abordagem coletiva sanitária, através de uma assistência integral e contínua a todos os membros das famílias da população adscrita à unidade de saúde, em cada uma das fases de seu ciclo de vida, apontando, assim, para a construção de um efetivo vínculo entre os enfermeiros, as famílias e a comunidade.

E este vínculo proporciona a enfermagem retornar a visão sistêmica e integral do indivíduo em seu contexto familiar e social, desenvolvendo ações de promoção, de proteção específica, de diagnóstico precoce, de rápida limitação do dano de recuperação da saúde e de reabilitação. Trabalhando com as reais necessidades locais, por meio de uma prática apropriada, humanizada e tecnicamente competente, sincronizando o saber popular com o saber técnico científico, em um verdadeiro encontro de gente cuidando de gente.

O modelo de atenção à saúde que os enfermeiros brasileiros estão ajudando a construir necessita de profissionais que permanentemente ampliem seus conhecimentos, apropriando-se das novas teorias e práticas. Eles devem estar sempre abertos para enfrentar novas experiências, identificar novos problemas críticos de sua realidade de trabalho, refletir sobre tais problemas e buscar, de forma interdisciplinar, soluções apropriadas para eles. Enfim, eles devem estar sempre disponíveis para o processo de ensino-aprendizagem.

Comparativamente, podemos dizer que o sistema de saúde, para o novo modelo, precisa muito mais do que músicos clássicos, que tocam cada nota de acordo com uma configuração predeterminada e preestabelecida, ele necessita de improvisadores do jazz que, uma vez tendo decidido que canção tocar, pegam sensivelmente as deixas um do outro e, na base disso, associado à interação com o espectador, decidem que notas tocar. A música é bela, inovadora e sempre adequada e construída para cada situação. E estes eventos e ou feitos "inesperados", "adversos" não inibem os enfermeiros que atuam no PSF a permanecerem à frente, na luta pelos seguintes investimentos:

- na articulação entre as instituições voltadas para a formação, capacitação e educação permanente de recursos humanos referentes à estratégia de Saúde da Família. A referida articulação 
vem se dando entre as Instituições de Ensino Superior e as Secretarias Estaduais e Municipais de Saúde. Trata-se, portanto, de uma rede de instituições comprometidas com a integração ensino/ serviço e voltada para o desenvolvimento da Estratégia de Saúde da Família no âmbito do SUS.

- na progressiva expansão exitosa da estratégia de saúde da família, em âmbito nacional, atingindo, nos dias de hoje, cerca de 10.473 equipes de saúde, em 3.090 municípios, vinculando-se a mais de 36 milhões de brasileiros.

- o desenvolvimento de um sistema de informação que fosse capaz de instrumentalizar os profissionais e gestores de saúde para o monitoramento e a avaliação da atenção básica por resultados de saúde, tornando-se um importante instrumento de planejamento estratégico, com vistas a tomada de decisões em tempo oportuno.

- na realização de pesquisas aplicadas ao PSF, a exemplo da Avaliação da Implantação e Funcionamento do Programa de Saúde da Família - PSF, (abr. - jul./1999), do Perfil dos Médicos e Enfermeiros do PSF - (ago. - set./1999), a Avaliação Qualitativa de Programas Sociais Prioritários, do governo federal (mar. - out./1998), "A Reforma do Sistema de Saúde no Brasil e o Programa Saúde da Família", coordenada pelo Professor Mário Dal Poz, da Universidade Estadual do Rio de Janeiro e, ainda, a avaliação do Programa Saúde da Família no Ceará, realizada pela Secretaria Estadual de Saúde, sob coordenação da Dra Maria Francisca Vieira de Andrade.

- no financiamento da atenção básica, bem como na sua forma de repasse, lembrando que até 1997, o Ministério da Saúde financiava os principais procedimentos da assistência básica prestados nos municípios através do pagamento direto pelos serviços prestados, ou seja, os prestadores recebiam pela realização de consultas médicas gerais, exames de pré natal, visitas de agentes comunitários de saúde, atendimentos de enfermagem, aplicação de vacinas, atendimentos odontológicos simples, entre outros.

A partir de 1998 essa lógica foi modificada. Criou-se o Piso de Atenção Básica - PAB, garantindo para financiamento do conjunto de procedimentos básicos o valor mínimo anual per capita de $R \$$ 10,00. Tal situação, mais que a garantia de um volume mínimo de recursos, assegurou também o repasse mensal, regular e automático diretamente ao fundo municipal de saúde, permitindo que o gestor municipal assuma, de fato, a responsabilidade pela prestação de serviços em seu município. Ele deixou de ser um prestador e passou a ser um gestor. Além da criação do Piso da Atenção Básica, definiu-se por incentivos, cujos recursos foram destinados à programas prioritários de atenção básica: Agentes Comunitários - PACS, Saúde da Família - PSF, Combate às Carências Nutricionais PCCN, Assistência Farmacêutica e Ações Básicas de Vigilância Sanitária.

Todos nós sabemos que não foram fáceis estas conquistas, uma vez que os conflitos, as discussões e os enfrentamentos cederam lugar ao diálogo permanente, a capacidade de persuação e a persistência de propósito, marcando uma característica impar dos Enfermeiros que atuam no PSF: a abertura das mentes e dos corações destes profissionais em busca da saúde para todas as famílias brasileiras.

\section{O PSF PAUTANDO NOVOS DESAFIOS À RECONSTRUÇÃO DA PRÁTICA NA ENFERMAGEM}

Há mais de vinte anos, em Alma-Ata, diversos países afirmaram a necessidade e a possibilidade de se atingir a saúde para todos no ano 2.000. Estamos as suas portas e, lamentavelmente, grande parte da população mundial está à margem dos sistemas de saúde, mantendo-se, ainda, uma chocante desigualdade no estado de saúde dos povos. Apontava, também, para a atenção primária como sendo composta de cuidados essenciais de saúde baseados em "métodos e tecnologias práticas, cientificamente bem fundamentadas e socialmente aceitáveis, colocadas ao alcance universal de indivíduos e famílias da comunidade, mediante sua plena participação", devendo representar o "primeiro nível de contato dos indivíduos, da família e da comunidade com o sistema nacional de saúde, e constituírem o primeiro elemento de um continuado processo de assistência à saúde".

Há 13 anos, em Ottawa, como resposta à importante demanda mundial para uma nova concepção de saúde, realizou-se a Primeira Conferência Internacional sobre Promoção da Saúde, destacando os progressos alcançados decorrentes da Declaração de Alma-Ata. Afirma que "para promover a saúde 
deve-se ir além do mero cuidado da mesma", devendo, a saúde, fazer parte da ordem do dia em todos os setores e em todos os níveis e que a responsabilidade pela promoção da saúde, por parte dos serviços de saúde, deve ser compartilhada entre os próprios indivíduos, os profissionais e gestores de saúde e os governos, ou seja, todos devem trabalhar em conjunto para se construir um sistema de proteção da saúde.

Dez anos depois, em Jacarta, na $4^{\text {a }}$ Conferência Internacional sobre Promoção da Saúde, realizada em 1997, foram estimuladas reflexões sobre os avanços alcançados até então e a identificação conjunta das direções e estratégias necessárias para o enfrentamento do desafio da promoção da saúde no século XXI.

Com vistas às ameaças emergentes à saúde, a Declaração de Jacarta aponta para a necessidade de novas ações, efetivas, concretas e capazes de destravar o potencial para a promoção da saúde existentes no indivíduo, na família, na comunidade e em diversos setores da sociedade organizada.

Associada aos eventos acima citados, a Carta do Caribe para a Promoção da Saúde, elaborada em 1993, durante a $13^{a}$ Reunião dos Ministros Responsáveis pela Saúde do Caribe, apontaram como estratégia para a concretização da promoção da saúde de seus povos, dentre outros importantes itens, a reorientação dos serviços de saúde.

Nos dias 18 e 19 de outubro do corrente ano, em Havana, Ministros da Saúde dos países IberoAmericanos reuniram-se para analisar e procurar consenso perante os problemas atuais relativos à saúde e, em especial, identificar possíveis vias alternativas relacionadas com os efeitos da globalização sobre o setor saúde. Dentre diversos pontos, destaca-se a afirmativa de que a estratégia fundamental para alcançar saúde para todos está constituída pela Atenção básica de Saúde, que garanta cobertura, acessibilidade, capacidade resolutiva e pertença a um sistema integrado de saúde.

Pelo exposto, a estratégia de Saúde da Família, recoloca na agenda nacional os desafios que os profissionais de saúde, neste caso a Enfermagem, terão pela frente, desafios estes que certamente os limite deste texto não me permite explorar um a um, todavia gostaria de lançar mão dos resultados apresentados na pesquisa Perfil dos Médicos e Enfermeiros que atuam no PSF, para ampliar o debate em torno dos seus principais achados:

- os enfermeiros atuam nos municípios do interior com menos de 50.000 habitantes das regiões Nordeste e Sudeste do País, com condições de trabalho a desejar;

do conjunto dos enfermeiros que atuam no PSF, 90,9\% são mulheres e com uma média de 34 anos; estes enfermeiros foram graduados em escolas de enfermagem de natureza pública $(70,7 \%)$, e têm em média 8 anos de formados;

- do universo total dos enfermeiros que atuam no PSF, "apenas" 37,1\% fizeram habilitação profissional, sendo a enfermagem em saúde pública, a obstétrica e a médico-cirúrgica as especialidades escolhidas na habilitação; somente 5,5\% fizeram um curso de especialização; 19,2\% têm título de especialista; 65,3\% têm acesso a publicações relativas à Saúde da Família, principalmente através de material editado pelo Ministério da Saúde;

- no geral, os enfermeiros sentem necessidade de aprimoramento profissional, sendo os cursos de especialização e o mestrado/doutorado escolhidos como forma de aprimoramento;

a área de atuação desenvolvida por 33,5\% é em enfermagem em saúde pública e dentro das unidades de saúde;

- o tempo de atuação no PSF é de menos de um ano $(44,0 \%)$, sendo selecionados para trabalhar, principalmente, através de entrevista $(35,6 \%)$ e contratados através de contrato temporário $(38,9 \%)$;

- a carga horária semanal dedicada ao trabalho é de 35 a 40h, e para isso auferem uma renda média de US $\$ 1.123,00$;

possuem outro vínculo empregatício (25,8\%), sendo este no setor público; e 17,3\% já atuaram no PSF de outro município;

- avaliam as condições de trabalho como desfavoráveis (62,0\%);

consideram a atividade profissional desgastante $(58,9 \%)$;

a relação entre os membros da equipe, contudo, é tida como boa/excelente $(83,2 \%)$ e o futuro do Programa é visto com otimismo por mais da metade destes profissionais. 
- Quero registrar, ainda, que os intensos esforços para garantia dos avanços institucionais e gerenciais que o PSF vem imprimindo no Brasil, só terá de fato sustentabilidade, se pactuarmos, negociarmos, estabelecermos acordos para superação dos problemas acima mencionados. E para tanto, exige vontade cooperativa e decisão das partes (gestores federal, estaduais e municipais). Caso contrário, a estratégia do PSF tenderá a se atomizar em milhares de modelos de atenção à saúde, reproduzindo os velhos males do sistema: ineficiência, ineq́uidade, pouca resolutividade, insatisfação profissional e das famílias/comunidades.

Finalmente, temos a responsabilidade de lutar pelo maior de todos os desafio, o compromisso com a ética da esperança, a ética do futuro, que começa hoje, na urgência de pagar a dívida com a sociedade brasileira: $\mathrm{o}$ direito de ter direito à saúde para todas as famílias.

\section{BIBLIOGRAFIA}

ALMEIDA, M.C.P.; MISHIMA, S.M.; SILVA, M.E.; MELLO, DF O trabalho de enfermagem e sua articulação com o processo de trabalho em saúde coletiva: rede básica de saúde. In: ALMEIDA, M.C.P. \& ROCHA, S.M.M (org.) O trabalho de enfermagem. São Paulo: Cortez, 1997.

ALMEIDA, M.C.P. de. O trabalho de enfermagem e sua articulação com o processo de trabalho em saúde coletiva: rede básica de saúde em Ribeirão Preto. Ribeirão Preto, 1991. Tese (Livre Docência) - Escola de Enfermagem de Ribeirão Preto, Universidade de São Paulo.

ALMEIDA, M.C.P. \& ROCHA, J.S.Y. O saber da enfermagem e sua dimensão prática. São Paulo: Cortez, 1986.

BRASIL. Ministério da Saúde. Saúde da família: uma estratégia de organização dos serviços de saúde. Ministério da Saúde. Secretaria de Assistência à Saúde: Brasília-DF, 1996.

BRASIL. Ministério da Saúde. Cadernos de Atenção Básica. Programa de Saúde da Família. Ministério da Saúde. Secretaria de Políticas de Saúde: Brasíli-DF, 2000.

CORDEIRO,H. Sistema Único de Saúde. Rio de Janeiro: Ayuri,1991.

CORDONI JÚNIOR, L. Medicina Comunitária: emergência e desenvolvimento na sociedade brasileira. São Paulo, 1979E.

DALMASO, A.S.W. Oferta e consumo de ações de saúde: como realizar o projeto de integralidade. Saúde em Debate, n. 44, p. 35-38, 1994.

FOUCAULT, M. O nascimento do hospital. In: FOUCAULT, M. Microfísica do poder. 5.ed. Rio de Janeiro: Graal, 1985.

HABERMAS, J. Consciência moral e agir comunicativo. Rio de Janeiro: Tempo Brasileiro, 1989.

NOVAES , H. de M. Ações integradas nos sistemas locais de saúde-SILOS: análise conceitual e apreciação de programas selecionados na América Latina. São Paulo: PROHASA, 1990.

PEREIRA, I.M.T.B.; PENTEADO, R.Z.; MARCELO, V.C. Promoção da saúde e educação em saúde: uma parceria saudável. O Mundo da Saúde, v. 24, n. 24, p. 39-43, 2000.

SILVA, G.B. Enfermagem profissional: análise crítica. São Paulo: Cortez, 1986.

VILLA, T.C.S.; MISHIMA, S.M.; ROCHA, S.M.M. A enfermagem nos serviços de saúde pública do Estado de São Paulo. In: ALMEIDA, M.C.P. \& ROCHA, S.M.M. (org.) O trabalho de enfermagem. São Paulo: Cortez, 1997.

VASCONCELOS, E.M. A priorização da família nas políticas públicas de saúde. Saúde em Debate, v.23, n.53, p.6-19, 1999. 